\title{
Los fanfictions como escritura en colaboración: modelos de lectores beta
}

\author{
Fanfictions as collaborative writing: \\ Models of beta readers
}

\author{
Anastasio García-Roca
}

Cómo citar este artículo:

García-Roca, Anastasio (2019). "Los fanfictions como escritura en colaboración: modelos de lectores beta”. El profesional de la información, v. 28, n. 4, e280404.

https://doi.org/10.3145/epi.2019.jul.04

Artículo recibido el 22-10-2018

Aceptación definitiva: 21-02-2019

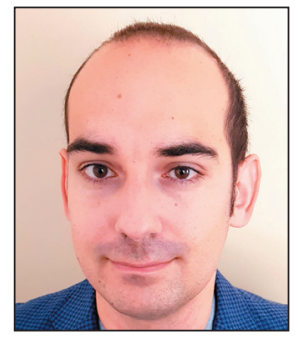

\author{
Anastasio García-Roca $\bowtie$ \\ https://orcid.org/0000-0002-2277-3034 \\ Universidad de Almería \\ Edificio Departamental de Humanidades \\ y Ciencias de la Educación II \\ Carretera Sacramento, $\mathrm{s} / \mathrm{n}$. \\ 04120 Almería, España \\ agr638@ual.es
}

\section{Resumen}

Dadas las potencialidades formativas y lúdicas que poseen las nuevas prácticas letradas desarrolladas en entornos digitales y, concretamente, los fanfictions, en este trabajo se han analizado 321 lectores beta (lectores que ayudan a mejorar los textos antes de su publicación) de la plataforma fanfiction.net. En estos perfiles se han podido conocer características generales como el sexo, edad y país de residencia, así como las fortalezas, debilidades y preferencias textuales que exponen estos beta readers. Todo ello ha permitido explorar las interacciones que se establecen entre escritores y sus lectores beta. Además, se han extraído cinco modelos de colaboración de lectores beta. Finalmente se ha comprobado que se trata de oportunidades únicas para el desarrollo de competencias lectoras, escritoras y literarias en escenarios informales.

\section{Palabras clave}

Fanfictions; Fics; Lectura; Lectores; Lectores beta; Escritura creativa; Aprendizaje; Educación literaria; Narrativa transmedia; Fandom; Cultura participativa.

\begin{abstract}
Given the formative and playful potential of the new literate practices developed in digital environments and, specificaIly, fanfictions, 321 beta readers of the fanfiction. net platform have been analyzed in this work. In the readers' profiles we have been able to know general characteristics such as sex, age or country of residence. As well as the strengths, weaknesses, textual preferences that these beta readers expose. All this has allowed us to explore the interactions that are established between writers and their beta readers. In addition, five collaboration models of beta readers have been extracted. Finally, it has been proven that these are unique opportunities for the development of reading, writing and literary skills in informal settings.
\end{abstract}

\section{Keywords}

Fanfictions; Fics; Reading; Readers; Beta readers; Creative writing; Learning; Literary education; Transmedia narrative; Fandom; Participative culture.

\section{Financiación}

Esta investigación forma parte del proyecto I+D titulado La lectura en la era digital: nuevas prácticas lectoras, cultura participativa y espacios de afinidad (ref. EDU2015-69924-R) del Ministerio de Economía y Competitividad dentro del Programa Estatal de I+D+i Orientado a los Retos de la Sociedad. 


\section{Introducción}

En el ámbito de la lectura literaria, la era digital ha permitido la evolución de nuevas textualidades basadas en las posibilidades tecnológicas que ofrecen los nuevos dispositivos. Destacan obras creadas para ser reproducidas en nuevos soportes con diferentes e innovadores modos de interactividad (respuesta táctil y realidad virtual y aumentada). Sin embargo, no es aquí donde hallamos la verdadera revolución de la lectura introducida por las nuevas tecnologías.

La lectura de libros en España sigue siendo mayoritariamente en formato códice sin innovaciones tecnológicas (FGEE, 2018). No obstante, no es tanto la naturaleza del texto lo que ha cambiado, como los procesos de recepción textual. Es decir, se lleva a cabo una lectura digital de obras convencionales.

Podríamos poner los ejemplos de las sagas literarias de Harry Potter (J. K. Rowling), Crepúsculo (Stephenie Meyer), Canción de hielo y fuego (George R. R. Martin) o, en el caso español, Memorias de Idhum (Laura Gallego). Es cierto que con el tiempo acabaron convirtiéndose en narrativas transmediáticas. Sin embargo, desde un principio la lectura que se ha realizado de estas obras no ha sido la convencional o clásica. En todos los ejemplos citados, internet ha jugado un papel central.

Esto se debe a la aparición de una cultura de la participación o cultura participativa (en términos de Jenkins, 2008) en la que se han desdibujado (al menos, en parte) las barreras para la expresión ciudadana. Se apoya la creatividad y la puesta en común de creaciones propias y colectivas de manera que los usuarios confían en que sus contribuciones son relevantes y sienten una conexión entre lo que dicen los demás y sus propias aportaciones (Aparici; Osuna-Acebo, 2013). Por tanto, conceptos como el de audiencia, comunicación de masas o medio necesariamente han de ser actualizados o revisados (Scolari, 2016; Scott, 2015).

Internet ha catalizado esta cultura de la participación (Jenkins, 2009). En la esfera de la lectura literaria, tanto en la selección como en la recepción, la Red suele estar presente (FGEE, 2018). La lectura literaria se ha convertido en una actividad social, especialmente para los adolescentes seguidores de la literatura infantil y juvenil (LIJ) (Lluch, 2014). Prueba de ello son la aparición de booktubers, redes sociales temáticas dedicadas a la lectura y escritura, de etiquetas (o hashtags) de LIJ y de infinidad de fanfictions. En este sentido, las aportaciones de Rovira-Collado (2017) apuntan a que estos elementos adquieren un incipiente protagonismo en la promoción de la LIJ. Parece que al menos un sector de la población vive la lectura atendiendo a la afirmación de García-Galera y Fernández-Muñoz (2016, p. 1): “si lo vives, lo compartes". Desde esta perspectiva, satisfacer esa necesidad de vivir socialmente o compartir las aficiones, intereses $u$ objetivos de las personas es mucho más fácil e inmediato en la era de las redes. De hecho, así es como se generan las grandes comunidades virtuales: por la unión de internautas en espacios de afinidad (Gee, 2004).

El entusiasmo generado en torno a conceptos como cultura participativa o produser no debe ser entendido como una respuesta activa, creativa y productiva por parte del lectorado en general (Van-Dijk, 2009; Schäfer, 2011). En este trabajo se entiende participación en su sentido más amplio con objeto de incluir a los participantes silentes o acechadores, es decir, aquellos usuarios que no participan explícitamente en el desarrollo de nuevos contenidos pero que su existencia se evidencia en el número de lecturas, visualizaciones, seguidores o en el mismo posicionamiento web (Guerrero-Pico, 2014b; Nielsen, 2006). Esto dificul-

ta el análisis de comunidades virtuales. Por ello seguimos la propuesta de Gee y Hayes (2011) de centrar el análisis en los espacios virtuales. Esto permite además analizar las huellas que los usuarios dejan y las peculiaridades o especificidades de estos escenarios virtuales.

En este trabajo se pretende realizar un acercamiento a estas nuevas prácticas letradas en su contexto natural (fanfiction. net), con objeto de conocer con mayor profundidad el proceso real de interacción entre escritores y lectores beta. Para ello se han perseguido los siguientes objetivos:

- Extraer las principales características sociodemográficas de los lectores beta.

- Analizar la experiencia y recorrido en la plataforma de los beta readers como lectores y escritores de fics.

- Explorar las fortalezas, debilidades y preferencias textuales que exponen los lectores beta en sus perfiles.

- Elaborar y describir los modelos de lectores beta existentes.

La consecución de los objetivos propuestos permitirá conocer, en su globalidad, estos nuevos alfabetismos desarrollados en contextos informales como prácticas vernáculas de los jóvenes y adolescentes.

\section{Revisión teórica}

La cultura participativa es un requisito previo para el desarrollo de narrativas transmediáticas en las que no sólo los contenidos se dispersan en diferentes medios y plataformas, sino que una parte de los usuarios adquieren el protagonismo 
en la creación de nuevas ampliaciones narrativas (Scolari, 2013). Entre estas extensiones destacan los fanfictions, definidos como manifestaciones culturales en las que los usuarios se apropian de textos y juegan con ellos expandiéndolos, modificándolos o rellenando posibles huecos argumentales (García-Roca, 2016). Todo esto posibilita a los seguidores expandir la experiencia de recepción de sus universos ficcionales preferidos.

En este fenómeno dejan de ser relevantes los derechos de autor y la propiedad intelectual ya que no existe un fin lucrativo. Desde esta perspectiva, es destacable el trabajo de The Organization for Transformative Works, una organización sin ánimo de lucro creada por y para los fans. Su intención es proteger legalmente, preservar y compartir las obras transformativas elaboradas por los usuarios (Coppa, 2014).

Podemos distinguir grosso modo dos grandes tipos de contenidos en la narrativa transmedia:

- oficiales;

- creados por los fans.

Scolari (2013) denomina canon a los contenidos oficiales protegidos por derechos de autor en los que priman las lógicas comerciales. Guerrero-Pico define canon como

"el universo o mundo diegético oficial (acontecimientos, personajes, coordenadas espaciales y temporales) desarrollado a través de todas las extensiones transmedia oficiales" (Guerrero-Pico, 2014b, p. 257).

En cambio, el fandom (fan + kingdom) es el reino de los fans: sus producciones, miembros, actividades, creencias, comentarios e interpretaciones. La combinación e interacción de ambos elementos permite la creación de narrativas transmedia.

Desde la investigación educativa, el fandom y especialmente los fanfictions han estado en el centro de numerosas investigaciones de reconocidos académicos (Black, 2008; Cassany, 2012; Knobel; Lankshear, 2006). Todos ellos han destacado el valor formativo que poseen estos nuevos alfabetismos en la educación no formal de los jóvenes y adolescentes. Desde este punto de vista los fanfictions son producciones realizadas en tiempos de ocio como prácticas vernáculas (Cassany, 2010) y forman parte de la vida personal de los escritores (Lankshear; Knobel, 2008; Barnes, 2015). En cualquier caso, esta forma de producción hipertextual se basa en la escritura colaborativa o social y requiere de desarrolladas competencias lectoras y literarias debido a que se debe conocer en profundidad la diégesis del hipotexto (texto origen) y además, competencias escritoras para la elaboración de las historias.

Se generan de este modo estructuras (auto)organizadas de colaboración basadas en la retroalimentación de los usuarios. En los fanfictions, y escritura creativa digital en general, encontramos no sólo a los escritores y sus lectores, sino que aparecen figuras híbridas como comentadores, calificadores, difusores, diseñadores gráficos y audiovisuales y lectores beta. Esta idea de colaboración abierta distribuida (o crowdsourcing), inteligencia colectiva o colabo-

Esta forma de producción hipertextual se basa en la escritura colaborativa y social

ración horizontal voluntaria o desinteresada obliga a replantear la idea tradicional de autor (Fathallah, 2017; Scott, 2015).

Para esta investigación nos hemos centrado en los lectores beta de fanfictions, ya que son usuarios que se ofrecen a ayudar en la corrección, narración, traducción o elaboración, en general, de las historias. Guerrero-Pico define lector beta como aquel

"fan que tiene acceso a borradores de fics para editarlos y sugerir cambios a los autores antes de su publicación" (Guerrero-Pico, 2014a, p. 729).

Esto es especialmente relevante debido a que este tipo de textos se suelen publicar de forma periódica y a medida que se van escribiendo: por semanas, meses u otros intervalos. Esta regularidad también está relativamente impuesta por los seguidores de las historias quienes solicitan la actualización de los contenidos para dar continuidad a la trama.

La misma web fanfiction.net ofrece una definición de lector beta:

"Un lector beta (o betalector, o beta) es una persona que lee una obra de ficción con ojos críticos, con el objetivo de mejorar la gramática, la ortografía, la caracterización y el estilo general de una historia antes de su presentación al público en general" https://www.fanfiction.net/betareaders

El lector beta, más que un editor o evaluador, es un claro ejemplo de audiencia prospectiva y participativa. Es decir, es un acompañante que ve el trabajo de manera más integral, centrado en la continuidad, defectos lógicos, etc. (Coppola, 2015). Desde esta perspectiva, no se establece jerarquía entre escritor y sus lectores beta, sino una interacción entre pares basada en una retroalimenta-
En el fanfiction se generan estructuras (auto)organizadas de colaboración basadas en la retroalimentación de los usuarios 
ción constructiva (Olin-Scheller; Wikström, 2010). La estrecha colaboración y la alta implicación de los lectores beta en el proceso de creación, favorece el desarrollo de vínculos afectivos y de amistad entre autores y lectores beta (Guerrero-Pico, 2015).

Por otro lado, tal y como se ha comentado anteriormente, la escritura de fanfictions requiere un dominio de la diégesis del hipotexto (en términos de Genette, 1989). En ocasiones, el universo ficcional es prácticamente inabarcable (por ejemplo, el multiverso Marvel). Para ello y aplicando el ideal de inteligencia colectiva (Lévy, 2004) y la creación colectiva (Casacuberta, 2003), los lectores beta se ofrecen para proteger el canon y evitar incoherencias respecto al hipotexto. Especialmente, en la plataforma se tratan de evitar los out of character ${ }^{1}$. En los alternative universe (AU) se desarrolla el fic con la intención explícita de explorar ucronías, crossovers y otras transformaciones de la diégesis que desafían al canon. No obstante, el fanfiction es una práctica caractercéntrica (Guerrero-Pico, 2014a) por lo que en cualquiera de los casos los personajes deben mantener su canonicidad. Guerrero-Pico (2015, p. 213) los denomina "guardianes del canon", puesto que "velan por la representación canónica de los personajes en el fanfiction".

Muchos autores jóvenes empiezan a escribir en solitario; otros en cambio se motivaron a hacerlo cuando descubrieron plataformas de escritura creativa como la de fanfiction. En cualquier caso, se trata de una respuesta natural a la cultura participativa que describe Henry Jenkins (2008). El itinerario de aprendizaje, en este sentido, es personal y construido por los usuarios (Siemens, 2004). Además, la existencia de mentores, ayudantes, colaboradores, aconsejadores o como queramos denominar a los lectores beta permite, junto con los comentarios de los seguidores de los fanfictions, el desarrollo de una personal learning network (Warlick, 2009). El aprendizaje se basa en la práctica, esfuerzo y retroalimentación con el resto de usuarios.

\section{Materiales y métodos}

Para la consecución de los objetivos propuestos, y teniendo en cuenta la revisión bibliográfica realizada, se ha optado por una investigación descriptiva ya que se ha tratado de

"observar a individuos, grupos, instituciones, métodos o materiales con el fin de describir, comparar, contrastar, clasificar, analizar e interpretar las entidades y los acontecimientos que constituyen sus diversos campos de investigación" (Cohen; Manion, 1990, p. 101).

Para la selección de la muestra se ha seguido un muestreo aleatorio simple que se basa en el principio de equiprobabilidad (Bisquerra-Alzina, 2016) con objeto de realizar una estadística descriptiva e inferencial en la que la muestra ha sido seleccionada a partir de la población global, de tal modo que todos los miembros de la población tienen la misma probabilidad de resultar escogidos (McMillan; Schumacher, 2005). La muestra contiene participantes con características similares a las de la población como un todo que permite reducir la varianza externa. La selección aleatoria permite al investigador generalizar los resultados más allá del grupo inmediato estudiado ya que ayuda al control del error asociado a características de sujetos en diferentes grupos (McMillan; Schumacher, 2005).

La muestra ( $n=321$ ) ha sido tomada del universo objeto de análisis conformado por todos los lectores beta que colaboran con los textos escritos en español $(n=1.141)$. El tamaño muestral responde a la saturación de los datos durante el proceso de análisis cualitativo. Se ha optado por fanfiction. net ${ }^{2}$ debido a que es el espacio virtual dedicado a la escritura hipertextual más grande de la Red (medido por el número de usuarios y de textos almacenados). Por poner un ejemplo, Harry Potter posee 780.000 fanfics sólo en esta web.

Para la triangulación de los datos, no sólo se ha analizado la ficha de lector beta, sino también su perfil de usuario en la plataforma, sus historias publicadas y, en determinados casos redes sociales que poseían perfil público. Esto ha permitido tomar datos desde diferentes fuentes de información con objeto de mejorar la validez y fiabilidad del estudio.

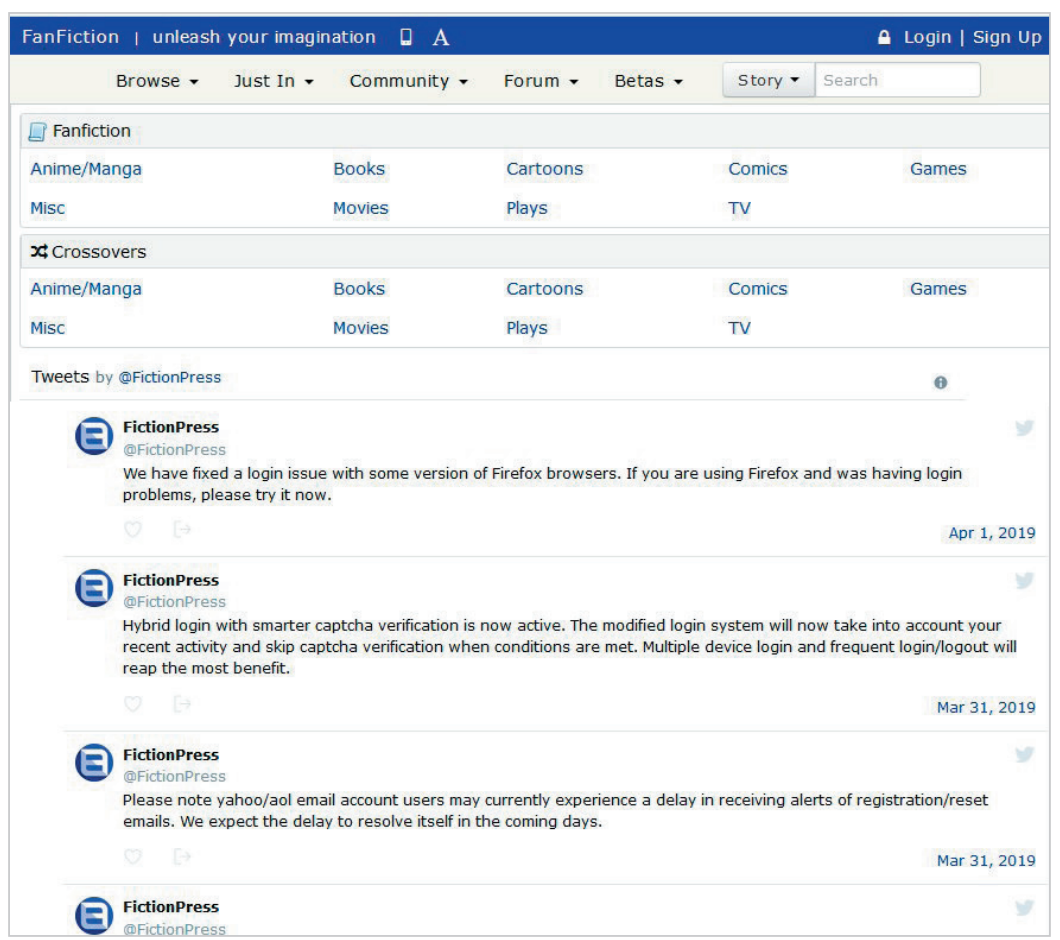

https://www.fanfiction.net 
En estas fichas de lectores beta, los usuarios deben exponer sus fortalezas y debilidades como editores, revisores o asesores. Para ello, se debe realizar un ejercicio metacognitivo y metalingüístico en el que se autoevalúen las habilidades expresivas y las competencias lectoras, escritoras y literarias. Por otro lado, deben exponer sus preferencias temáticas, de género, mediales, así como el nivel de la clasificación del contenido (también denominado de madurez). Finalmente deben incluir el idioma en el que prefieren betear. Además se han extraído datos como sexo, edad de inicio en la plataforma y actual, país de residencia y número de historias escritas.

Toda esta información ha sido procesada, codificada y categorizada con el paquete informático de análisis de datos Nvivo 12. Las categorías han sido traspasadas al programa SPSS 23 para su análisis cuantitativo. Aunque el análisis cualitativo se fundamentó en una codificación emergente, se partió de las categorías y conclusiones de investigaciones precedentes (Guerrero-Pico, 2014a; Black, 2008), que fueron ampliadas y modificadas.

\section{Resultados}

\subsection{Características sociodemográficas}

En primer lugar se analizaron los datos sociodemográficos con objeto de conocer el perfil de los lectores beta de fanfiction.net. El tamaño de la muestra y la clara diferencia existente nos permite afirmar que la plataforma es fundamentalmente femenina a nivel poblacional. El $85,9 \%$ de los que se ha podido extraer su sexo son chicas frente al $14,1 \%$ de chicos.

Estos usuarios tienen una edad comprendida entre 9 y 40 años $(R=31)$, aunque la media obtenida es 19,47 años. Es destacable que la mediana se sitúa en 19 años. Si comparamos estos datos con la edad de inicio en la plataforma, hallamos que estos lectores beta empezaron en fanfiction.net con una media de 15,17 años.

Es decir, en general estos usuarios llevan más de 4 años en la plataforma (el $86,4 \%$ tienen entre $1 \mathrm{y}$ 6 años de experiencia en la web). También es destacable que el tercer cuartil (Q3) de la edad de inicio se sitúa en 17 años, por tanto el $75 \%$ de los lectores beta empezaron siéndolo en edad escolar según el sistema educativo español. De media los usuarios se registraron en la plataforma a mediados de 2012.

En cuanto a la distribución geográfica, se han tomado datos de fanfictioners procedentes de hasta 27 países diferentes. A pesar de que son lectores beta de fanfictions en español, el 45,2\% de los perfiles son estadounidenses. En segundo lugar destacan México y España que representan un $14,9 \%$ y un $10,4 \%$ de la muestra respectivamente. Finalmente aparecen otros países como Argentina $(6,6 \%)$, Chile $(4,1 \%)$, Colombia (2,9\%), Venezuela (2,5\%) e Inglaterra (2,1\%). Esto está relacionado con el idioma en el que tienen publicado sus perfiles: a pesar de que se filtraron los datos para analizar a usuarios que betean hipertextos

Tabla 1. Distribución de la muestra en función del sexo

\begin{tabular}{|l|l|c|c|c|}
\cline { 3 - 5 } \multicolumn{2}{c|}{} & Frecuencia & Porcentaje & Porcentaje válido \\
\hline \multirow{3}{*}{ Válido } & Chico & 29 & 9,0 & 14,1 \\
\cline { 2 - 5 } & Chica & 177 & 55,1 & 85,9 \\
\cline { 2 - 5 } & Total & 206 & 64,2 & 100 \\
\hline Perdidos & Sistema & 115 & 35,8 & \\
\hline Total & 321 & 100 & \\
\hline
\end{tabular}

Tabla 2. Distribución de la muestra en función de la edad de inicio, actual y el tiempo en la plataforma

\begin{tabular}{|c|c|c|c|c|}
\hline & & Edad de inicio & Edad actual & $\begin{array}{c}\text { Tiempo en la } \\
\text { plataforma }\end{array}$ \\
\hline \multicolumn{2}{|l|}{ Media } & 15,17 & 19,47 & 4,27 \\
\hline \multicolumn{2}{|l|}{ Mediana } & 15,00 & 19,50 & 4,00 \\
\hline \multicolumn{2}{|c|}{ Desviación estándar } & 3,845 & 4,503 & 2,424 \\
\hline \multicolumn{2}{|l|}{ Varianza } & 14,781 & 20,275 & 5,875 \\
\hline \multirow{3}{*}{ Percentiles } & 25 & 13,00 & 16,00 & 2,00 \\
\hline & 50 & 15,00 & 19,50 & 4,00 \\
\hline & 75 & 17,00 & 22,00 & 5,00 \\
\hline \multirow{2}{*}{$n$} & Válidos & 86 & 86 & 81 \\
\hline & Perdidos & 235 & 235 & 238 \\
\hline
\end{tabular}

Tabla 3. Distribución de la muestra en función del país de residencia

\begin{tabular}{|c|c|c|c|c|}
\hline & & \multirow{3}{*}{$\begin{array}{c}\text { Frecuencia } \\
1\end{array}$} & \multirow{3}{*}{$\begin{array}{c}\text { Porcentaje } \\
0,3\end{array}$} & \\
\hline & & & & \multirow{2}{*}{$\begin{array}{c}\begin{array}{c}\text { Porcentaje } \\
\text { válido }\end{array} \\
0,4\end{array}$} \\
\hline \multirow{11}{*}{ Válidos } & No especifica & & & \\
\hline & Estados Unidos & 109 & 34,0 & 45,2 \\
\hline & México & 36 & 11,2 & 14,9 \\
\hline & España & 25 & 7,8 & 10,4 \\
\hline & Argentina & 16 & 5,0 & 6,6 \\
\hline & Chile & 10 & 3,1 & 4,1 \\
\hline & Colombia & 7 & 2,2 & 2,9 \\
\hline & Venezuela & 6 & 1,9 & 2,5 \\
\hline & Inglaterra & 5 & 1,6 & 2,1 \\
\hline & Otros & 26 & 7,8 & 10,4 \\
\hline & Total & 241 & 75,1 & 100 \\
\hline Perdidos & Sistema & 80 & 24,9 & \\
\hline \multicolumn{2}{|l|}{ Total } & 321 & 100 & \\
\hline
\end{tabular}


de libros en español, el 60,7\% de los perfiles están escritos en inglés. Esto indica que los usuarios escriben y corrigen en otros idiomas, además del materno (leen y betean en al menos dos idiomas).

Además de ser un fenómeno internacional, también lo es multilingüe. De los perfiles analizados es destacable que tan solo el 68,5\% de los usuarios revisan textos únicamente en español. O lo que es lo mismo: al menos el $31,5 \%$ de los lectores beta se ofrecen para asesorar textos en dos o más idiomas. Recordemos además que el $45,2 \%$ de los perfiles analizados pertenecen a estadounidenses. Encontramos que el $17,1 \%$ del total lo hace además en inglés y un $6,5 \%$ en francés. En total se registran 12 segundos idiomas (entre los que se incluye el latín).

\subsection{Experiencia y preferencias textuales}

Estos usuarios no sólo colaboran en la redacción y narración, sino que en la mayoría de los casos son grandes lectores: tienen de media marcadas más de 153 historias como favoritas. Siguen a más de 31 autores. Todo ello indica que son lectores de una gran cantidad y variedad de fanfictions.

Hay que añadir que los usuarios analizados, además de desempeñar el rol de lectores y lectores beta, son también escritores de fanfictions. Estos usuarios tienen 11,8 historias de media publicadas en la plataforma. Hay que tomar estos datos con cierta prudencia debido a que algunos de los perfiles están sin actualizar mucho tiempo y es posible que los autores hayan dejado la plataforma y borrado sus textos. Por tanto, podrían ser incluso más.

Tabla 4. Estadísticos descriptivos de las historias escritas, favoritas y autores favoritos

\begin{tabular}{|c|c|c|c|c|}
\hline \multicolumn{2}{|c|}{ Experiencia lectora y escritora } & Historias escritas & Historias favoritas & Autores favoritos \\
\hline \multicolumn{2}{|c|}{ Media } & 11,80 & 153,11 & 31,59 \\
\hline \multicolumn{2}{|c|}{ Error estándar de la media } & 1,018 & 22,376 & 6,334 \\
\hline \multicolumn{2}{|c|}{ Mediana } & 7,00 & 41,00 & 9,00 \\
\hline \multicolumn{2}{|c|}{ Moda } & 6 & 1 & 0 \\
\hline \multicolumn{2}{|c|}{ Desviación estándar } & 18,178 & 398,390 & 112,603 \\
\hline \multicolumn{2}{|c|}{ Varianza } & 330,452 & 158714,704 & 12679,348 \\
\hline \multirow{2}{*}{$n$} & Válidos & 319 & 317 & 316 \\
\hline & Perdidos & 2 & 4 & 5 \\
\hline
\end{tabular}

La web no incluye un apartado en el que los usuarios puedan indexar sus redes sociales con objeto de compartir o promocionar sus textos entre sus allegados. A pesar de esto, el 18,4\% de los usuarios analizados referencian sus redes sociales: Twitter, Instagram, Facebook, Tumblr o YouTube.

Al tratarse de una actividad altruista y voluntaria, los lectores beta también incluyen cuáles son sus preferencias a la hora de betear y dedicar su tiempo. Éstas se basan en dos grandes apartados:

- el género $(89,1 \%)$ por ejemplo: aventura, humor, romance, etc.;

- el medio del hipotexto (14,6\%): libros, películas, videojuegos, anime, cómics, espectáculos, series televisivas o dibujos animados.

Por otro lado, en estos perfiles de presentación señalan aquellas obras con las que no colaborarían. El 43,3\% de la muestra rechaza textos de una temática específica: generalmente rehúsan textos basados en historias románticas centradas en una relación o un amor romántico (novelas rosa). El 11\% de estos usuarios concretan que no aceptarán textos en los que se desarrollen y se detengan en escenas de sexo. Esto contrasta con la temática principal de la comunidad: de los 784.000 fanfictions de Harry Potter, 410.000 se incardinan en la categoría de "romance" (de las 23 existentes), lo que representa un 52,2\% del total; en el caso de Twilight es un $64,48 \%$ o, en el caso Percy Jackson and the Olympians, un $47,27 \%$.

En cuanto a la clasificación del contenido en función de la edad, es importante señalar que $97,5 \%$ de los usuarios betean textos clasificados en Rated $K$ (recomendado para todas las edades ya que no existe un lenguaje grosero, temas adultos o violencia). No son exigentes en este apartado, ya que el $14,6 \%$ restringe su colaboración a textos clasificados hasta la categoría Rated T (mayores de 13 años) y el 82,8\% hasta los clasificados en Rated $M$ (recomendado para mayores de 16 años debido a referencias a violencia, lenguaje grosero o contenido sexual).

En relación con la experiencia como lectores beta es reseñable que de media han beteado textos en más
Tabla 5. Estadísticos descriptivos de experiencia y oferta de géneros diferentes

\begin{tabular}{|c|c|c|c|}
\hline & & $\begin{array}{l}\text { Experiencia géne- } \\
\text { ros diferentes }\end{array}$ & $\begin{array}{c}\text { Oferta de géne- } \\
\text { ros diferentes }\end{array}$ \\
\hline \multicolumn{2}{|c|}{ Media } & 5,52 & 14,32 \\
\hline \multicolumn{2}{|c|}{ Mediana } & 5,00 & 15,00 \\
\hline \multicolumn{2}{|c|}{ Desviación estándar } & 3,359 & 4,853 \\
\hline \multicolumn{2}{|c|}{ Varianza } & 11,285 & 23,551 \\
\hline \multicolumn{2}{|c|}{ Mínimo } & 1 & 1 \\
\hline \multicolumn{2}{|c|}{ Máximo } & 21 & 21 \\
\hline \multirow{2}{*}{$n$} & Válidos & 318 & 310 \\
\hline & Perdidos & 3 & 11 \\
\hline
\end{tabular}


de 5 géneros. Por tanto el género no suele ser tampoco un filtro demasiado denso ya que en promedio los beta readers se exponen a colaborar con textos clasificados en 14 géneros. Esto indica que se ofrecen para revisar y corregir una gran variedad de textos de diferentes temas y géneros.

\subsection{Fortalezas, debilidades y modelos de lector beta}

A continuación se muestran los resultados de las fortalezas y debilidades que los lectores beta exponen en sus perfiles de usuarios. Las fortalezas (en este contexto) son los elementos que estos internautas, tras un ejercicio metacognitivo, metalingüístico y metaliterario, reconocen como sus cualidades principales en la ayuda en la producción de fanfics. Además exponen los aspectos en los que están dispuestos a colaborar. La codificación y posterior categorización dieron como resultado tres grandes bloques:

- Lector beta para el plano de la expresión (o relato) del texto que se divide en dos subcategorías: corrección textual y función poética;

- Lector beta para el plano de la traducción;

- Lector beta para el plano de contenido (o historia) con dos subcategorías: coherencia narrativa respecto al hipotexto y construcción de nuevos contenidos.

Mantendremos en nuestro análisis la dicotomía forma-contenido, si bien es cierto que son dos aspectos de todo producto artístico-literario inseparables e interdependientes.

\subsubsection{Plano de expresión (o relato)}

En este bloque, la función del lector beta es ayudar a mejorar y enriquecer la redacción mediante la detección de errores ortográficos, gramaticales y léxico-semánticos, así como de inconsistencias en el procedimiento estilístico. La labor de corrección lingüística ocupa el lugar preferente en este apartado de la plataforma virtual. Por eso ésta es la categoría más importante y frecuente de todo el análisis. Tanto es así que en la plataforma se relaciona la calidad de un fanfiction con escribir con precisión y corrección. El 74,8\% de los lectores beta analizados se centran en, entre otros elementos, adaptar el texto a las normas que regulan la correcta escritura de una lengua: gramática, ortografía, sintaxis, etc. Concretamente:

- el $56,7 \%$ del total expone que tiene un alto grado de competencia gramatical;

- el $57,9 \%$ destaca que puede ayudar con la ortografía en general;

- el 23,4\% se centra en signos de puntuación o acentuación en particular;

- un 11,8\% indica que puede ayudar en la coherencia y cohesión de la estructura general de la narración.

El análisis mixto de los datos ha permitido extraer un primer modelo de lector beta que asesora únicamente en los aspectos formales sin interferir en el desarrollo de la historia o del plano del contenido. Generalmente estos usuarios no restringen los fandoms o temas que betearán. Este modelo matiza e insiste en que tan sólo revisará aspectos formales del texto sin interferir en la trama o en aspectos estilísticos. Por tanto, la retroalimentación entre los escritores y lectores beta se limita a las interacciones propias de corrección textual.

Por ejemplo, algunos de ellos señalan que si los escritores así lo desean y sólo en ese caso, se ofrecen a colaborar también en el desarrollo de la trama general de los fics. Sin embargo, el hecho de no especificar el fandom ni la temática/ género lo convierte en una de sus debilidades. Es decir, estos betareaders no son los más indicados para revisar la canonicidad del texto ni continuar la historia.

En la escritura creativa no sólo se valora la corrección lingüística, sino también el uso de la lengua literaria. Atraer la atención del lector sobre la forma del mensaje verbal conlleva extraer su máxima potencialidad expresiva mediante la aplicación de estrategias estilísticas. Desde esta perspectiva, otra fortaleza a la que hacen referencia los lectores beta es la ayuda a embellecer el estilo del relato. En esta categoría se ha incluido una gran variedad de códigos relacionados con la narración y los recursos literarios: figuras retóricas, dominio del repertorio léxico, fortaleza del nivel connotativo del lenguaje, transformación de oraciones y otros elementos. Debe indicarse que el $19,6 \%$ de los participantes comentan que poseen un alto desarrollo de la competencia léxico-semántica lo que les ha permitido mejorar la calidad poética de los textos beteados. El 8,1\% se ofrece para mejorar el relato detectando inconsistencias en el procedimiento

Tabla 6. Frecuencia absoluta y relativa en fortaleza en aspectos formales (FAF)

\begin{tabular}{|l|c|c|}
\cline { 2 - 3 } \multicolumn{1}{c|}{} & Frecuencia & Porcentaje \\
\hline Gramática & 182 & 56,7 \\
\hline Ortografía & 186 & 57,9 \\
\hline Puntuación & 75 & 23,4 \\
\hline Coherencia textual & 38 & 11,8 \\
\hline Total & 240 & 74,8 \\
\hline
\end{tabular}

Tabla 7. Frecuencia absoluta y relativa de fortaleza en aspectos estilísticos (FAE)

\begin{tabular}{|l|c|c|}
\cline { 2 - 3 } \multicolumn{1}{c|}{} & Frecuencia & Porcentaje \\
\hline Varios elementos & 42 & 13,1 \\
\hline Metáforas & 2 & 0,6 \\
\hline Variedad léxica & 63 & 19,6 \\
\hline Función poética & 26 & 8,1 \\
\hline Total & 104 & 32,4 \\
\hline
\end{tabular}


estilístico y sugiriendo recursos retóricos posibles, etc. Si nos atenemos al discurso, es decir a cómo se desarrolla la historia, es reseñable que un 13,1\% expone que su fortaleza reside en la ambientación, la construcción y la caracterización y evolución de los personajes, así como la consistencia del conflicto o de los hechos narrados, entre otros.

De lo anteriormente expuesto se infiere un segundo modelo de lector beta, que colabora con el escritor para mejorar la calidad estética o función poética del texto. Estos betareaders comienzan a participar en el proceso de creación textual. Asimismo la relación que se establece sigue siendo desigual: los lectores beta sugieren cambios superficiales en la manera en que el autor relata los hechos, sin intervenir en el desarrollo de la historia.

Son usuarios con un mayor nivel de exigencia en sus colaboraciones: rechazan textos con errores formales. En general este modelo no especifica el fandom, aunque sí la temática del fic: romance, drama, terror, aventuras, etc. Son usuarios que disfrutan con la narración, recreación y descripción de escenas concretas. Sin embargo, no pueden ampliar la historia debido a que posiblemente no conocen el hipotexto.

\subsubsection{Plano de la traducción}

Por otra parte, los escritores desean que sus historias sean leídas por el máximo número de usuarios y tengan un notorio alcance internacional. Para ello es fundamental eliminar fronteras como pueden ser los idiomas. Hay un $13,1 \%$ de lectores beta que indica que la traducción es uno de sus puntos fuertes. Se trata de una actividad en la que primero se interpreta el texto origen para luego producir el nuevo en otra lengua. Indican que tienen buena competencia traductora, que les permite solucionar posibles problemas léxico-semánticos, morfo-sintácticos, pragmáticos, retóricos y culturales en el proceso de traslación.

Se ha hallado por tanto un tercer modelo de lector beta que se centra en cuestiones específicas de traducción, ya sea revisar o realizar la traducción completa del fic. Se trata de una posición híbrida Tabla 8. Frecuencia absoluta y relativa en fortaleza como traductor (FT)

\begin{tabular}{|l|c|c|}
\cline { 2 - 3 } \multicolumn{1}{c|}{} & Frecuencia & Porcentaje \\
\hline Traducción & 42 & 13,1 \\
\hline
\end{tabular}
que se sitúa entre la competencia traductora y mediadora. Estos usuarios intervienen directamente en el plano estilístico y formal del fic traducido. Además se ha comprobado que los lectores beta que colaboran en la traducción de fics ofrecen su asesoramiento en la revisión de aspectos formales en uno o ambos idiomas. Habitualmente no especifican fandom ni género.

Este modelo de lector beta es el más exigente de todos debido al esfuerzo que requiere la traducción de un fic. Dependiendo de la proporción de la traducción, la nueva versión puede pasar a formar parte del perfil del traductor reconociendo la autoría del escritor. Destacan que su principal debilidad es el tiempo que se demoran en la traslación.

\subsubsection{Plano del contenido}

En esta categoría se incluyen elementos relacionados con el desarrollo narrativo de los fanfictions. El $53 \%$ de los lectores beta analizados señala que su fortaleza se relaciona con este bloque. Los escritores de fanfictions deben dominar a la perfección la diégesis del hipotexto, es decir, el mundo ficticio en el que se desarrolla la historia del canon. El 24\% afirma que puede asesorar en la caracterización de los personajes. Desde esta perspectiva, el 10,6\% detalla que su ayuda evitará los out of character (OOC), esto impedirá que el personaje trazado en el fanfiction sea endeble e inconsistente con respecto a la manera de actuar, pensar o hablar en el texto de origen o hipotexto. Éste es uno de los elementos más sensibles en la elaboración de un fic. Existe una aversión generalizada hacia las transformaciones excesivas de los personajes, ya que restan credibilidad y, en opinión de los fanfictioners, calidad al fic. La canonicidad de los textos reside especialmente en los personajes. Esto lo podemos observar en los universos alternos (alternative universes) y crossovers en los que se modifica total o parcialmente la diégesis manteniendo intactos a los personajes. Finalmente, un $22,1 \%$ de los lectores beta analizados asesora en varios elementos relacionados con el desarrollo narrativo.

Otros usuarios reconocen que tienen un alto desarrollo de la competencia genérica y se ofrecen como asesores y revisores de las reglas específicas de cada (sub)género literario o temática: romances, tragedias, fantasía, humor, poesía, aventuras, etc. De hecho, el 18,1\% de la muestra se ofrece para betear géneros concretos.

En esta ocasión se pueden extraer dos modelos de lectores beta:

- usuarios que velan por mantener la canonicidad de fanfiction para evitar los OOC. Desde esta óptica sí que especifican qué fandoms dominan y cuáles están dispuestos a betear, ya que deben identificar incoherencias narrativas del fanfic respecto al hipotexto. Las interacciones que potencialmente se establezcan entre escritor y lector beta se centran en debates relacionados con la historia original;

- aquel que se ofrece para co-crear una historia, salvar un bloqueo del autor o continuar un fanfic iniciado. La relación 
que se establece entre los protagonistas es la más horizontal de todos los modelos extraídos. El beta reader colabora con el autor original, y sus sugerencias no sólo afectan al plano de la expresión, sino que interviene directamente en el desarrollo global del fanfic.

Paradójicamente, una fortaleza y a la vez debilidad que señalan los usuarios que se encuentran en este último modelo es la especialización en una temática concreta. Es decir, publican qué temáticas dominan y cuáles son las que no serían capaces de continuar.
Se han hallado cinco funciones de los lectores beta: corregir, sugerir, traducir, debatir y colaborar

Las características de los cinco modelos de lector beta se recogen en la tabla 10.

Tabla 10. Resumen de los modelos de lectores beta

\begin{tabular}{|c|c|c|c|c|c|}
\hline $\begin{array}{l}\text { Modelos de } \\
\text { lector beta }\end{array}$ & Planos & Objeto & Fandom & Tema/género & Rol \\
\hline Modelo 1 & \multirow{2}{*}{ Plano de la expresión } & Aspectos formales & \multirow{3}{*}{ No especifica } & No especifica & Corrige \\
\hline Modelo 2 & & Función poética & & Especifica & Sugiere \\
\hline Modelo 3 & Plano de la traducción & Traducción & & \multirow{2}{*}{ No especifica } & Traduce \\
\hline Modelo 4 & \multirow{2}{*}{ Plano del contenido } & Fidelidad al hipotexto & \multirow{2}{*}{ Especifica } & & Debate \\
\hline Modelo 5 & & Continúa el fanfiction & & Especifica & Colabora \\
\hline
\end{tabular}

\section{Discusión y conclusión}

El análisis de los resultados nos ha permitido comprobar que el lectorado beta es mayoritariamente femenino y con una edad media de estar cursando Educación Secundaria Obligatoria (ESO) según el sistema educativo español, aspecto que coincide con las conclusiones de investigaciones de Jenkins (2008), Guerrero-Pico (2014a) y Hellekson (2009). Se trata igualmente de un fenómeno de alcance internacional y multilingüe en el que no son aplicables barreras geográficas. De igual modo, concuerda con los resultados de la reconocida académica Black (2008), quien concluye que los autores de fanfictions son jóvenes y adolescentes de diferentes países.

Los usuarios permanecen varios años y evolucionan dentro de la plataforma, se hacen más exigentes, se convierten en tutores, asesores o lectores beta. Este hecho se relaciona con las conclusiones de la investigación longitudinal de Lammers y Marsh (2018), quienes documentan la evolución de una escritora de fanfics y muestran que la escritura creativa forma parte de su identidad duradera en el tiempo.

A pesar de esto no existen requisitos para ser lector beta, pero se ha comprobado que éstos cuentan con cierta experiencia dentro de la plataforma. Son lectores de una gran cantidad de (hiper)textos de autores y temáticas diversas, pero también poseen experiencia en la escritura de fanfiction. Por tanto, se trata de usuarios con conocimientos y competencias suficientes en la plataforma para actuar como mentores en todo el proceso de creación de fanfictions. Estos resultados coinciden con los obtenidos por Campbell y otros (2016), que analizaron la tutoría distribuida que se lleva a cabo en comunidades de fans online.

Desde esta perspectiva, se han identificado cinco modelos de beta readers que se especializan en diferentes apartados del proceso de creación textual: aspectos formales, de traducción y de contenido. Esto refuerza la idea de beta reader como experto en un área.

En cuanto a las fortalezas y debilidades, destacan los aspectos formales de la narración ya que, al tratarse de escritores jóvenes y adolescentes, existen ciertas dificultades en este sentido. Por otro lado, los betareaders también ayudan en aspectos relacionados con los elementos narrativos y desarrollo de la historia. El escritor de fanfictions ideal debe conocer todos los detalles de la diégesis del hipotexto. Pero cuando esto no sucede, los lectores beta ayudan en el desarrollo de personajes, relaciones, escenarios o tramas. Esto coincide con la definición de Guerrero-Pico (2015), quien considera a los beta readers como guardianes del canon, puesto que son los encargados de revisar el texto para mantener la canonicidad de los contenidos y no alejarse demasiado del mundo narrativo previo.

Los fanfictioners pueden tener varios lectores beta que les asesoren en elementos específicos de sus textos. Se hace complicado la delimitación de roles diferenciados: los beta readers, además de colaboradores, son también escritores en la plataforma y lectores. Es decir, los usuarios desempeñan diferentes papeles al mismo tiempo. De este modo se crea una estructura autoorganizada basada en la retroalimentación y colaboración entre los usuarios: esto es uno de los elementos más importantes que estimula el funcionamiento de la plataforma. En ella se almacenan millones de fanfictions, se reúnen varios miles de lectores beta, incontables escritores e infinidad de lectores. En ningún caso existe 
un fin lucrativo, sino que todos los esfuerzos se basan en una motivación para el ocio o intrínseca. A esta misma conclusión se llega en investigaciones precedentes como la elaborada por Evans et al. (2017) sobre la misma plataforma de escritura de fanfiction. Se trata además de una plataforma que cumple las características que definen los espacios de afinidad descritos por Gee (2004).

A estas alturas es indudable el enorme interés y fascinación que despierta la ficción amateur o fan en los jóvenes. Si a esto le añadimos las potencialidades formativas y educativas que posee todo el proceso de creación y recepción textual, convierte a esta práctica letrada vernácula en una oportunidad única para la realización de propuestas de innovación educativa. Por ello se hace necesario imbricar contextos de enseñanza y escenarios de aprendizaje formal, no formal e informal (Scolari; Lugo-Rodríguez; Masanet, 2019).

Para acabar, el presente estudio abre nuevas líneas de investigación:

- ¿Qué relación existe entre los lectores beta, sus textos y sus colaboraciones?

- ¿Cómo son las interacciones y el intercambio de borradores entre los escritores de fics y sus betas?

- ¿Cómo se podrían integrar estos aprendizajes en la educación formal?

\section{Notas}

1. Los out of character $(O O C)$ son errores narrativos en los que se desarrolla una transvalorización excesiva de un personaje que se aleja demasiado del canon. En cambio, cuando los personajes actúan siguiendo el canon se conoce como in character (IC).

2. Fanfiction.net no es el único espacio de reunión de fanfictioners: también destacan otros como LiveJournal, ArchiveofOurOwn o Wattpad. Cada uno posee características propias y reúnen a comunidades diferentes.

\section{Referencias}

Aparici, Roberto; Osuna-Acebo, Sara (2013). "La cultura de la participación”. Mediterranean journal of communication, v. 4, n. 6, pp. 137-148.

https://doi.org/10.14198/MEDCOM2013.4.2.07

Barnes, Jennifer L. (2015). "Fanfiction as imaginary play: What fan-written stories can tell us about the cognitive science of fiction". Poetics, v. 48, pp. 69-82.

https://doi.org/10.1016/j.poetic.2014.12.004

Bisquerra-Alzina, Rafael (2016). Metodología de la investigación educativa. Madrid: La Muralla. ISBN: 9788471337481

Black, Rebecca W. (2008). Adolescents and online fan fiction. New York: Peter Lang. ISBN: 9780820497389

Campbell, Julie-Ann; Aragon, Cecilia; Davis, Katie; Evans, Sarah; Evans, Abigail; Randall, David P. (2016). "Thousands of positive reviews: distributed mentoring in online fan communities". In: Gergle, Darren; Morris, Meredith R. Proceedings of the $19^{\text {th }}$ ACM Conf on computer-supported cooperative work \& social computing. New York: ACM, pp. $691-704$.

https://arxiv.org/abs/1510.01425

https://doi.org/10.1145/2818048

Casacuberta, David (2003). Creación colectiva. En internet el creador es el público. Barcelona: Gedisa. ISBN: 97884 74329285

Cassany, Daniel (2010). "Leer y escribir al margen de la ley". En: Cilelij. I Congreso iberoamericano de lengua y literatura infantil y juvenil. Actas y memoria del congreso. Madrid: Fundación SM/ Ministerio de Cultura de España, pp. 497-514. ISBN: 8435240516578

https://www.academia.edu/7130732/Leer_y_escribir_literatura_al_margen_de_la_ley

Cassany, Daniel (2012). En_línea. Leer y escribir en la Red. Barcelona: Anagrama. ISBN: 9788433933966

Cohen, Louis; Manion, Lawrence (1990). Métodos de investigación educativa. Madrid: La Muralla. ISBN: 9788471335654

Coppa, Francesca (2014). "Pop cultre, fans, and social media”. In: Hunsiger, Jeremy; Senft, Theresa M. The social media handbook. London: Routledge. ISBN: 9780415886802

Coppola, Brian P. (2015). "Book and media recommendations: Stories, style, and a few study breaks". Journal of chemical education, v. 92, n. 7, pp. 1140-1142.

https://doi.org/10.1021/acs.jchemed.5b00359

Evans, Sarah; Davis, Katie; Evans, Abigail; Campbell, Julie; Randall, David; Yin, Kodlee; Aragon, Cecilia (2017). “More than peer production: Fanfiction communities as sites of distributed mentoring". In: Lee, Charlotte; Poltrock, Steve. CSCW'17 Procs of the 2017 ACM Conf on computer supported cooperative work and social computing. New York: ACM, pp. $259-272$. https://doi.org/10.1145/2998181.2998342 
Fathallah, Judith M. (2017). Fanfiction and the author. How fanfic changes popular cultural texts. Amsterdam: Amsterdam University Press. ISBN: 9789089649959

FGEE (2018). Hábitos de lectura y compra de libros en España 2017. Madrid: Federación de Gremios de Editores de España.

http://federacioneditores.org/img/documentos/HabitosLecturaCompraLibros2017.pdf

García-Galera, María-del-Carmen; Fernández-Muñoz, Cristóbal (2016). Si lo vives, lo compartes. Cómo se comunican los jóvenes en un mundo digital. Barcelona: Ariel. ISBN: 9788408159933

https://www.fundaciontelefonica.com/arte_cultura/publicaciones-listado/pagina-item-publicaciones/itempubli/499

García-Roca, Anastasio (2016). "Prácticas lectoras en espacios de afinidad: formas participativas en la cultura digital". Ocnos: Revista de estudios sobre lectura, v. 15, n. 1, pp. 42-51.

https://doi.org/10.18239/ocnos_2016.15.1.979

Gee, James-Paul (2004). Situated language and learning. A critique of traditional schooling. New York: Routledge. ISBN: 0203309642

http://networkedlearningcollaborative.com/wp-content/uploads/2015/07/james-paul-gee-situated-language-andlearning-a-critique-of-traditional-schooling-2004.pdf

Gee, James-Paul; Hayes, Elisabeth R. (2011). Language and learning in the digital age. New York: Routledge. ISBN: 978 0415602778

Genette, Gerard (1989). Palimpsestos: la literatura en segundo grado. Altea: Taurus. ISBN: 9788430621958

Guerrero-Pico, Mar (2014a). "Producción y lectura de fan fiction en la comunidad online de la serie Fringe: transmedialidad, competencia y alfabetización mediática". Palabra clave, v. 18, n. 3, pp. 722-745.

https://doi.org/10.5294/pacla.2015.18.3.5

Guerrero-Pico, Mar (2014b). “Webs televisivas y sus usuarios: un lugar para la narrativa transmedia. Los casos de 'Águila Roja' y 'Juego de Tronos' en España”. Comunicación y sociedad, n. 21, pp. 239-267.

http://comunicacionysociedad.cucsh.udg.mx/index.php/comsoc/article/view/578

Guerrero-Pico, Mar (2015). Historias más allá de lo filmado: Fan fiction y narrativa transmedia en series de televisión. Tesis doctoral. Barcelona: Universitat Pompeu Fabra.

https://repositori.upf.edu/handle/10230/26921

Hellekson, Karen (2009). “A fannish field of value: Online fan gift culture”. Cinema journal, v. 48, n. 4, pp. 113-118. https://doi.org/10.1353/cj.0.0140

Jenkins, Henry (2008). Convergence culture: la cultura de la convergencia de los medios de comunicación. Barcelona: Paidós. ISBN: 9788449321535

Jenkins, Henry (2009). Fans, blogueros y videojuegos. La cultura de la colaboración. Barcelona: Paidós. ISBN: 97884 49322587

Knobel, Michelle; Lankshear, Colin (2006). "Digital literacy and digital literacies: Policy, pedagogy and research considerations for education". Nordic journal of digital literacy, v. 1, pp. 12-24.

https://www.idunn.no/dk/2006/01/digital_literacy_and_digital_literacies_-_policy_pedagogy_and_research_cons

Lammers, Jayne C.; Marsh, Valerie L. (2018). "A writer more than... a child”: A longitudinal study examining adolescent writer identity. Writter communication, v. 35, n. 1, pp. 89-114.

https://doi.org/10.1177/0741088317735835

Lankshear, Colin; Knobel, Michelle (2008). Nuevos alfabetismos. Su práctica cotidiana y el aprendizaje en el aula. Madrid: Morata. ISBN: 9788471125200

Lévy, Pierre (2004). Inteligencia colectiva: por una antropología del ciberespacio. Washington DC: Organización Panamericana de la Salud. ISBN: 2707126934

http://inteligenciacolectiva.bvsalud.org/public/documents/pdf/es/inteligenciaColectiva.pdf

Lluch, Gemma (2014). "Jóvenes y adolescentes hablan de lectura en la red". Ocnos: Revista de estudios sobre lectura, n. 11, pp. 7-20.

https://doi.org/10.18239/ocnos_2014.11.01

McMillan, James H.; Schumacher, Sally (2005). Investigación educativa. Madrid: Pearson. ISBN: 9788483226872

Nielsen, Jakob (2006). "The 90-9-1 rule for participation inequality in social media and online communities". Nielsen Norman Group, 9 October.

https://www.nngroup.com/articles/participation-inequality 
Olin-Scheller, Christina; Wikström, Patrik (2010). "Literary prosumers: Young people's reading and writing". Education inquiry, v. 1, n. 1, pp. 41-56.

https://doi.org/10.3402/edui.v1i1.21931

Rovira-Collado, José (2017). "Booktrailer y booktuber como herramientas LIJ 2.0 para el desarrollo del hábito lector". Investigaciones sobre lectura, n. 7, pp. 55-72.

http://rua.ua.es/dspace/handle/10045/62755

Schäfer, Mirko-Tobias (2011). Bastard culture! How user participation transforms cultural production. Amsterdam: Amsterdam University Press. ISBN: 9789089642561

http://mtschaefer.net/media/uploads/docs/Schaefer_Bastard-Culture_2011.pdf

Scolari, Carlos A. (2013). Narrativas transmedia: Cuando todos los medios cuentan. Barcelona: Deusto. ISBN: 97884234 13362

Scolari, Carlos A. (2016). "El translector. Lectura y narrativas transmedia en la nueva ecología de la comunicación". En: Millán, José-Antonio. La lectura en España. Informe 2017. Madrid: Federación de Gremios de Editores de España, pp. 175-186. ISBN: 9788486141615

https://dialnet.unirioja.es/servlet/articulo?codigo $=5803284$

Scolari, Carlos A.; Lugo-Rodríguez, Nohemi; Masanet, Maria-José (2019). “Educación transmedia. De los contenidos generados por los usuarios a los contenidos generados por los estudiantes". Revista latina de comunicación social, n. 74, pp. 116-132.

https://doi.org/10.4185/RLCS-2019-1324

Scott, Suzanne (2015). "The moral economy of crowdfunding and the transformative capacity of fan-ancing". New media and society, v. 17, n. 2, pp. 167-182.

https://doi.org/10.1177/1461444814558908

Siemens, George (2004). "Connectivism: A learning theory for the digital age". Internacional journal of instructional technology \& distance learning, v. 2, n. 1.

https://writix.co.uk/blog/connectivism-a-learning-theory-for-the-digital-age

Van-Dijk, José (2009). "Users like you? Theorizing agency in user-generated content". Media culture and society, v. 31, n. 1, pp. 41-58.

https://doi.org/10.1177/0163443708098245

Warlick, David (2009). “Grow your personal learning network: New technologies can keep you connected and help you manage information overload". Learning \& leading with technology, v. 36, n. 6, pp. 12-16.

La Fundación Biblioteca Social es una institución sin ánimo de lucro que se constituyó en el año 2014. Tiene como objetivo contribuir a compensar los desequilibrios sociales apoyando proyectos que llevan a cabo las bibliotecas públicas, dirigidos a los sectores más vulnerables de la sociedad.

\section{¿Colaboras?}

fundacionbibliotecasocial.org info@fundacionbibliotecasocial.org

3 @Biblio_Social

f FundacionBibliotecasSocial

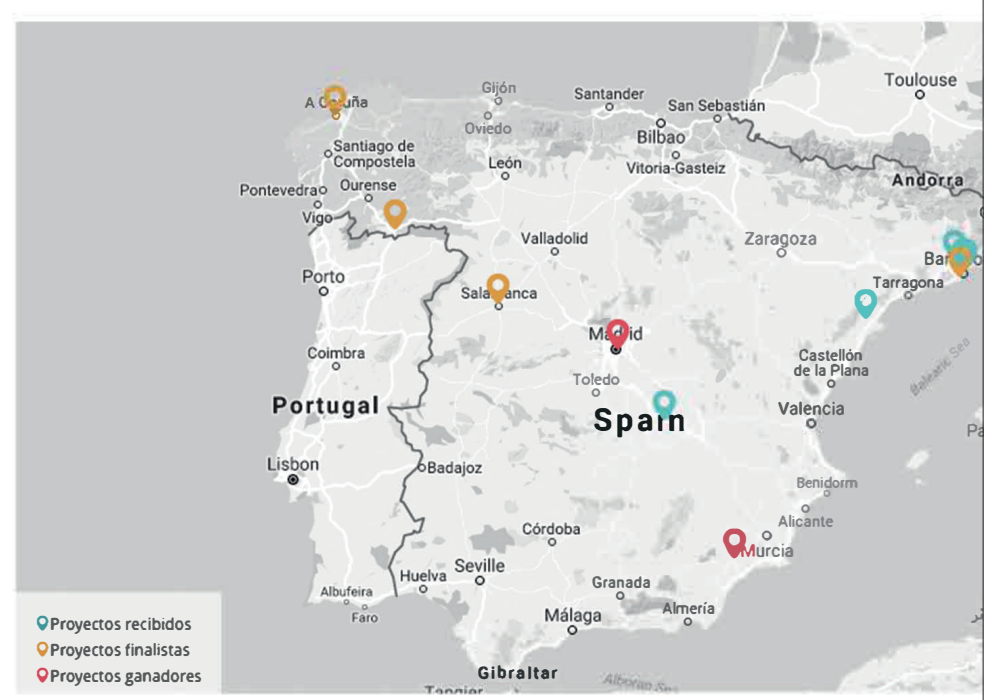

Mapa de proyectos de bibliotecas públicas para la inclusión social. 2016

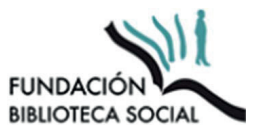

